\title{
Methodological Aspects of Lactate Measurement - Evaluation of the Accuracy of Photometric and Biosensor Methods
}

\author{
A. Biedler ${ }^{1}$, S. Schneider ${ }^{2}$, F. Bach ${ }^{3}$, S. Soltész ${ }^{4}$, W. Wilhelm ${ }^{5}$, S. Ziegeler ${ }^{2}$ and S. Kreuer ${ }^{*}, 2$ \\ ${ }^{1}$ Kath. Kliniken Essen-Nord, Marienhospital Altenessen, Hospitalstrasse 24, D-45329, Essen, Germany \\ ${ }^{2}$ Klinik für Anaesthesiologie, Intensivmedizin und Schmerztherapie, Universitätsklinikum des Saarlandes, D-66421, \\ Homburg/Saar, Germany \\ ${ }^{3}$ Klinik für Anaesthesiologie und operative Intensivmedizin, Krankenanstalten Gilead, D-33617, Bielefeld, Germany \\ ${ }^{4}$ Klinik für Anaesthesiologie und operative Intensivmedizin, Klinikum Leverkusen, D-51375, Leverkusen, Germany \\ ${ }^{5}$ Klinik für Anästhesiologie und operative Intensivmedizin, St. Marienhospital, D-44534, Lünen, Germany
}

\begin{abstract}
Introduction: The present investigation was designed to investigate the accuracy and precision of lactate measurement obtained with contemporary biosensors (Chiron Diagnostics, Nova Biomedical) and standard enzymatic photometric procedures (Sigma Diagnostics, Abbott Laboratories, Analyticon).

Materials and Methods: Measurements were performed in vitro before and after the stepwise addition of 1molar sodium lactate solution to samples of fresh frozen plasma to systematically achieve lactate concentrations of up to $20 \mathrm{mmol} / \mathrm{l}$.

Results: Precision of the methods investigated varied between $1 \%$ and $7 \%$, accuracy ranged between $2 \%$ and $-33 \%$ with the variability being lowest in the Sigma photometric procedure (6\%) and more than $13 \%$ in both biosensor methods.

Conclusion: Biosensors for lactate measurement provide adequate accuracy in mean with the limitation of highly variable results. A true lactate value of $6 \mathrm{mmol} / \mathrm{l}$ was found to be presented between 4.4 and $7.6 \mathrm{mmol} / \mathrm{l}$ or even with higher difference. Biosensors and standard enzymatic photometric procedures are only limited comparable because the differences between paired determinations presented to be several mmol. The advantage of biosensors is the complete lack of preanalytical sample preparation which appeared to be the major limitation of standard photometry methods.
\end{abstract}

Keywords: Biosensor, photometry, substrate specific electrode, plasma samples, standard laboratory procedures, accuracy, precision, in vitro.

\section{INTRODUCTION}

Contemporary lactate analysis is based either on standard photometric techniques or on recently introduced biosensors. Photometric techniques are typically applied in the clinical laboratory and therefore believed to provide high accuracy and reliability [1]. However, the turnaround time resulting from sample transport and preparation is often in contradiction to clinical demands, especially when repeated lactate measurements within a short period of time are necessary. Biosensors are usually integral part of so-called "blood gas analyzers", providing not only lactate but also a variety of other parameters (e.g. blood gases, $\mathrm{pH}$, electrolytes, hemoglobin, etc.) without delay on a $24 \mathrm{~h} /$ day basis. Moreover, blood gas analyzers require no preparation of the samples and can be operated easily at the point of care by nonspecialized staff. However, this non-professional operation is said to incorporate more sources of errors and inaccuracy compared to clinical laboratory determinations [2]. Several authors have addressed the analytical performance of biosensor devices by comparing their results with established

*Address correspondence to this author at the Klinik für Anaesthesiologie, Intensivmedizin und Schmerztherapie, Universitätsklinikum des Saarlandes, Kirrberger Str., D-66421, Homburg/Saar, Germany; Tel: +49 6841 1622443; E-mail: sascha.kreuer@uniklinikum-saarland.de laboratory methods [2-5]. However, comparative studies on that topic are limited since a "gold standard" for lactate measurement is not available and all procedures are known to be significantly influenced by methodological and sample specific factors. Furthermore, blood samples drawn from patients will provide only limited information regarding the accuracy of measurements of high lactate levels over 10 $\mathrm{mmol} / \mathrm{l}$. Therefore, the present investigation was performed in vitro in order to achieve systematic information on the accuracy, precision and comparability of lactate measurement obtained with contemporary biosensor and standard laboratory photometrical methods.

\section{MATERIALS AND METHODOLOGY}

\section{Analyzers}

Three standard photometric procedures, which were all performed in a specialized clinical laboratory were investigated: (1) no. 735 (Sigma Diagnostics, St. Louis, MO), (2) TDxFLx (Abbott Laboratories, Irving, TX), and (3) Lactat PAP (Analyticon, Burbach, Germany). The biosensor systems investigated were (1) series 800 (Chiron Diagnostics, Medfield, MA) and (2) STAT Profile 9 (Nova Biomedical, Waltham, MA), both operated by non-specialized staff. Quality control, system monitoring and analyzer mainte- 
nance were performed according to the recommendations of the manufacturers. Biosensors were replaced when indicated.

\section{Sample Material}

Lactate concentrations were measured in samples of thawed fresh frozen plasma provided by the local blood bank. We limited our investigation to the analysis of plasma samples because photometric detection - opposed to biosensors - requires the centrifugation of blood prior to analysis and results obtained in blood samples significantly differ from those measured in centrifuged plasma, especially when sample dilution due to high lactate concentrations is necessary [5].

\section{Experimental In Vitro Design}

The design of this investigation was based on a step by step addition of 1molar sodium lactate solution to plasma samples to systematically generate specimens with lactate concentrations of up to $20 \mathrm{mmol} / \mathrm{l}$. Two consecutive subsets of experiments were performed. In a first series, simultaneous measurements were performed with the biosensor analyzer of Nova Biomedical and photometric procedures of Sigma Diagnostics and Abbott Laboratories after sodium lactate was added to the plasma samples in steps of $5 \mathrm{mmol} / \mathrm{l}$ $(+5,+10,+15 \mathrm{mmol} / \mathrm{l})$. The second series consisted of measurements with the biosensors of Nova Biomedical and Chiron Diagnostics and the photometric Analyticon procedure after addition of sodium lactate in steps of $2 \mathrm{mmol} / \mathrm{l}(+2,+4$, $+6, \ldots,+16,+18 \mathrm{mmol} / \mathrm{l})$. Lactate concentrations were analyzed in duplicates before (blank value) and after addition of sodium lactate. Measurements with biosensors were performed every 3-5 minutes. All personal involved in analyzing was informed about the study but blinded for the lactate concentrations in the samples.

\section{Calculations}

Precision was determined as the repeatability in the duplicates' measurements. We calculated the the mean relation of the differences between duplicate values to the mean values of the duplicates:

Precision [\%]

$=$ mean of the sum of $\{($ value $1-$ value 2$) \times 100 /(($ value $1+$ value 2) / 2) $\}$

Accuracy was determined from the degree of agreement between the measured values and the expected values. We calculated the mean relation of the differences between measured values and expected values in relation to the expected values.

\section{Accuracy [\%] \\ $=$ mean of the sum of $\{$ (expected value - measured value $)$ / expected value $\mathrm{x} 100\}$}

For each analysis the expected value was calculated from the lactate concentration in the sample plus the amount of lactate added.

\section{Statistics}

Accuracy of lactate measurement was evaluated by repeated measures ANOVA, precision by Wilcoxon rank sum

\section{fresh frozen plasma (sample material)}

\begin{tabular}{|c|c|}
\hline $\begin{array}{l}\text { Addition of } 1 \text { molar sodium lactate solution } \\
\text { in steps of } 5 \mathrm{mmol} / \mathrm{l} \\
(+5,+10,+15 \mathrm{mmol} / \mathrm{l})\end{array}$ & $\begin{array}{l}\text { Addition of } 1 \text { molar sodium lactate solution } \\
\text { in steps of } 2 \mathrm{mmol} / 1 \\
(+2,+4,+6, \ldots,+16,+18 \mathrm{mmol} / \mathrm{l})\end{array}$ \\
\hline $\begin{array}{l}\text { Lactate measurement (in duplicates) with: } \\
\text { - standard photometric procedures: } \\
\text { - no. } 735 \\
\text { (Sigma Diagnostics, St. Louis, MO) } \\
\text { - TDxFLx } \\
\text { (Abbott Laboratories, Irving, TX) } \\
\text { - biosensor systems: } \\
\text { - STAT Profile } 9 \\
\text { (Nova Biomedical, Waltham, MA) }\end{array}$ & $\begin{array}{l}\text { Lactate measurement (in duplicates) with: } \\
\text { - standard photometric procedures: } \\
\text { - Lactat PAP } \\
\text { (Analyticon, Burbach, Germany) } \\
\text { - biosensor systems: } \\
\text { - series } 800 \\
\text { (Chiron Diagnostics, Medfield, MA) } \\
\text { - STAT Profile } 9 \\
\text { (Nova Biomedical, Waltham, MA) }\end{array}$ \\
\hline
\end{tabular}

Fig. (1). Flow diagram of the experimental design. 
test. Differences between the methods were evaluated using the unpaired two-tailed t-test for continuous variables or the Mann-Whitney test as indicated. Comparability was obtained using the Bland and Altman method of analysis where the bias is defined as the mean difference between two methods and the precision as the standard deviation of the mean difference $[6,7]$. All data are presented as mean and standard deviation. A $\mathrm{p}$ value less than 0.05 was considered statistically significant.

\section{RESULTS}

In total, 3 biosensors of Nova Biomedical (Nova 1-3) and 3 biosensors of Chiron Diagnostics (Chiron 1-3) were investigated. 600 measurements were performed in 10 series with steps of $2 \mathrm{mmol} / \mathrm{l}$ lactate (Nova Biomedical, Chiron Diagnostics, (biosensors); Analyticon (photometry)) and 120 measurements in 5 series with steps of $5 \mathrm{mmol} / \mathrm{l}$ lactate (Nova Biomedical (biosensor); Abbott Laboratories, Sigma Diagnostics (photometry)).

\section{Blank Values}

The mean lactate concentration in the samples before adding the sodium lactate solution was reported as $1.24 \pm$ $0.47 \mathrm{mmol} / \mathrm{l}$ (Nova Biomedical (biosensor)), $1.23 \pm 0.49$ $\mathrm{mmol} / \mathrm{l}$ (Chiron Diagnostics (biosensor)), $1.02 \pm 0.10 \mathrm{mmol} / \mathrm{l}$ (Sigma Diagnostics (photometry)), $1.05 \pm 0.17 \mathrm{mmol} / 1$ (Abbott Laboratories (photometry)), and $0.90 \pm 0.48 \mathrm{mmol} / \mathrm{l}$ (Analyticon (photometry)). Results differed statistically significant between all photometric methods and all biosensor analyzers, and between the procedures of Abbott Laboratories and Analyticon (all $\leq 0.05)$.

\section{Precision}

Precision was in total $2.1 \pm .2 .4 \%$ (Chiron Diagnostics (biosensor)), $2.5 \pm 2.9 \%$ (Nova Biomedical (biosensor)), 1.2 $\pm 0.7 \%$ (Sigma Diagnostics (photometry)), $7.1 \pm 8.4 \%$ (Abbott Laboratories (photometry)), and $1.0 \pm 1.1 \%$ (Analyticon (photometry)) (Table 1). The precision calculated for each single biosensor separately was $1.6 \pm 1.6 \%$ (Chiron 1), $5.2 \pm 6.3 \% \quad$ (Chiron 2), $1.7 \pm 1.3 \% \quad$ (Chiron 3) and $2.1 \pm 3.7 \%$ (Nova 1), $1.6 \pm 2.0 \%$ (Nova 2), $4.0 \pm 3.3 \%$ (Nova 3). All differences in duplicates were without statistical significance.

\section{Accuracy}

Mean accuracy of lactate measurement presented to be $1.7 \pm 13.4 \%$ (Chiron Diagnostics (biosensor)), $9.7 \pm 13.6 \%$ (Nova Biomedical (biosensor)), $3.4 \pm 5.7 \%$ (Sigma Diagnostics (photometry)), $-33.4 \pm 18.0 \%$ (Analyticon (photometry)), and $-38.4 \pm 18.6 \%$ (Abbott Laboratories (photometry)) (Table 1). The accuracy of every single biosensor calculated separately was $-1.6 \pm 11.5 \%$ (Chiron 1), $-21.0 \pm 21.7 \%$ (Chiron 2), $10.8 \pm 5.0 \%$ (Chiron 3) and $1.2 \pm 12.9 \%$ (Nova 1), $-11.8 \pm 16.2 \%$ (Nova 2), $22.1 \pm 19.4 \%$ (Nova 3). The accuracy obtained with the photometric Abbott Laboratories and Analyticon procedures had to be excluded from further interpretations, because after unblinding the results to the investigators at the end of the investigation, it became obvious that the laboratories had insufficiently diluted samples with lactate concentrations higher than $5 \mathrm{mmol} / \mathrm{l}$. These mis- takes remained undetected despite the performance of a quality control in the laboratories.

Table 1. Accuracy of Lactate Measurement by Method

\begin{tabular}{|c|c|c|c|}
\hline & \multicolumn{3}{|c|}{ Accuracy $[\%]$ in Lactate Concentration Ranges } \\
\hline & $0-20 \mathrm{mmol} / \mathrm{l}$ & $0-10 \mathrm{mmol} / \mathrm{l}$ & $10-20 \mathrm{mmol} / \mathrm{l}$ \\
\hline \multicolumn{4}{|c|}{ Biosensors } \\
\hline Chiron Diagnostics & $1.7 \pm 13.4$ & $6.2 \pm 10.5$ & $-2.0 \pm 15.7$ \\
\hline Nova Biomedical & $9.7 \pm 13.6$ & $12.9 \pm 12.6$ & $5.3 \pm 14.5$ \\
\hline \multicolumn{4}{|c|}{ Photometry } \\
\hline Sigma Diagnostics & $3.4 \pm 5.7$ & $2.4 \pm 3.4$ & $3.5 \pm 7.5$ \\
\hline Abbott Laboratories & $-33.4 \pm 18.0 *$ & $-20.7 \pm 16.3 *$ & $-40.9 \pm 15.5 *$ \\
\hline Analyticon & $-38.3 \pm 18.6 *$ & $-20.4 \pm 11.8 *$ & $-50.2 \pm 13.7 *$ \\
\hline
\end{tabular}

Measurements were performed before and after the stepwise addition of increasing amounts of sodium lactate to fresh frozen plasma samples. Presented are mean differences in percent between measured values and expected values (expected value $=$ lactate value measured before adding sodium lactate + amount of lactate solution added) separately shown in ranges of 0-20, 0-10 and 10-20 mmol/l.

Best accuracy was obtained with the photometric Sigma Diagnostics procedure. Both biosensor methods (Chiron Diagnostics, Nova Biomedical) were limited by the high variability of the results. The high inaccuracy of the photometric procedures of Abbott Laboratories and Analyticon was caused by undetected dilution errors of the laboratory.

Accuracy $[\%]=($ expected value - measured value $) /$ expected value $\mathrm{x} 100$.

Mean \pm standard deviation.

$* \mathrm{p}<0.05$ expected $v s$ measured value.

\section{Between-Method Comparison}

The degree of agreement between the simultaneously investigated biosensor methods of Nova Biomedical and Chiron Diagnostics and the photometric method of Sigma Diagnostics was estimated using the Bland and Altman analysis. The results regarding bias, precision, and the limit of agreement are shown in Table 2 and Fig. (2).

Table 2. Analysis of Agreement Between Different Methods of Lactate Measurement as Described by Bland and Altman

\begin{tabular}{|c|c|c|c|}
\hline & $\begin{array}{c}\text { Bias } \\
{[\mathbf{m m o l} / \mathbf{l}]}\end{array}$ & $\begin{array}{c}\text { Precision } \\
{[\mathbf{m m o l} / \mathbf{l}]}\end{array}$ & $\begin{array}{c}\text { Limits of Agreement } \\
\text { [mmol/l] }\end{array}$ \\
\hline \hline $\begin{array}{c}\text { Chiron Diagnostics } \\
v s \text { Nova Biomedical }\end{array}$ & -0.8 & 1.8 & $-4.4-2.8$ \\
\hline $\begin{array}{c}\text { Chiron Diagnostics } \\
\text { vs Analyticon }\end{array}$ & 3.6 & 3.3 & $-3.0-10.2$ \\
\hline $\begin{array}{c}\text { Nova Biomedical } \\
v s \text { Sigma Diagnostics }\end{array}$ & 1.2 & 1.0 & $-0.8-3.2$ \\
\hline
\end{tabular}

Calculated were the bias as the mean of the differences between paired determinations, the precision as the standard deviation of the mean difference (SD) and the limits of agreement as the mean differences \pm 2 SD from all split sample comparisons of the methods investigated.

Only modest agreement was found within the biosensor methods (Chiron Diagnostics, Nova Biomedical) and between the biosensor methods and the photometric procedure of Sigma Diagnostics showing that results obtained with different devices are only moderately interchangeable.

Bias $=$ mean of the differences between paired determinations

Precision $=$ standard deviation of the bias.

Limits of agreement $=$ mean difference \pm 2 SD .

\section{DISCUSSION}

The measurement of lactate concentration has developed as an integral portion of care for the critically ill patient [8, 


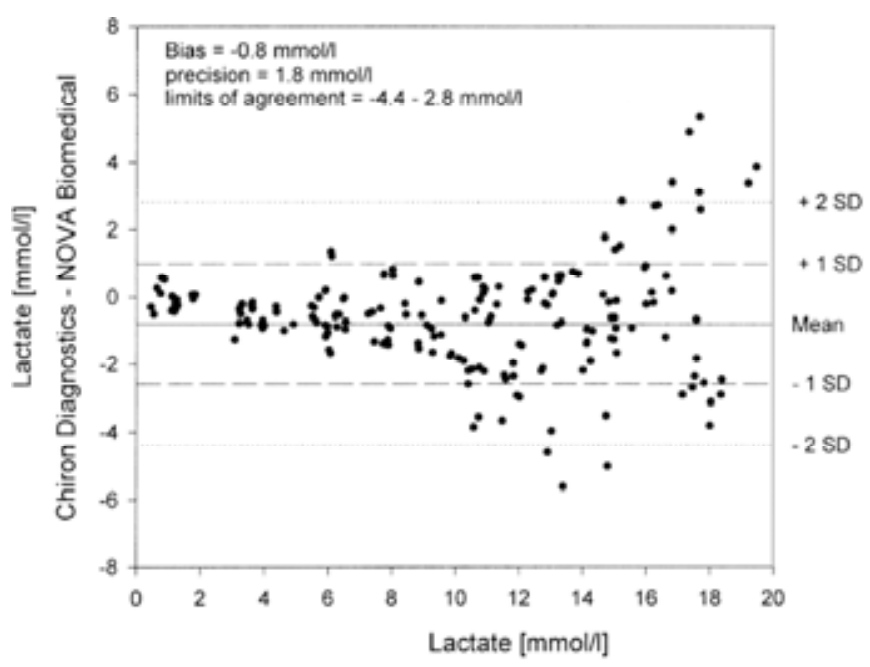

(Chiron Diagnostics - NOVA Biomedical) / 2

Bland and Altman analysis of the simultaneously split sample measurements with the biosensor methods of NOVA Biomedical and Chiron Diagnostics. Displayed are the mean difference $(0.8 \mathrm{mmol} / \mathrm{l})$, the precision $(1.8 \mathrm{mmol} / \mathrm{l})$ and the limits of agreement $(-4.4$ to $2.8 \mathrm{mmol} / \mathrm{l})$ in plasma samples with lactate concentrations from $0-20 \mathrm{mmol} / \mathrm{l}$.

The plot shows the high dispersion of the results especially in lactate concentrations of $10 \mathrm{mmol} / \mathrm{l}$ and more.

Fig. (2). Analysis of agreement between biosensor methods.

9]. Analysis is either performed with photometric-based techniques that are used in clinical laboratories, or with substrate specific biosensors that are implemented in point-ofcare analyzers. As laboratory procedures are subject to quality assurance programs, they are still stated as the "gold standard" for lactate measurement $[1,10]$, whereas biosensors are suggested to have the potential of more sources of errors [1]. Indeed, the best precision in our investigation was found with a photometric procedure (1.2\%, Sigma Diagnostics) but also the precision of both biosensor methods was at least acceptable $(2.1 \%)$ and for all procedures in the same order of magnitude as found by other investigators $[10,11]$. In contrast to the calculation of the precision, the accuracy of lactate measurement is more difficult to assess. Comparing a new device with an established one as done in other investigations only allows conclusions on the comparability of the results but not on their correctness [12]. In our study we tried to solve the problem of the missing reference method by performing the investigation in vitro. Sodium lactate was added to plasma samples to produce sample material with exactly predefined lactate concentrations (by calculating expected values out of the amount of lactate added and the blank lactate concentration in the sample before adding any lactate solution). Moreover, plasma was chosen as the carrier to avoid any influences on the results due to ongoing glycolysis or the gradient between plasma and red blood cells as already reported [13-15]. With this methodology, also another limitation could be bypassed. In most investigations samples were obtained from patients directly with the consequence of little or no information on the accuracy of lactate measurement in the concentration range above $10 \mathrm{mmol} / \mathrm{l}$. By adding increasing amounts of lactate solution it was possible to achieve lactate concentrations of up to $20 \mathrm{mmol} / \mathrm{l}$ and to cover the complete measuring range of the biosensor methods, which are said to detect lactate even in this high concentration without dilution. With respect to our results, best accuracy was obtained with the photometric Sigma procedure and the Chiron biosensors. A accuracy of $3.4 \%$ and $1.7 \%$ respectively in mean from samples with lactate concentrations of up to $20 \mathrm{mmol} / \mathrm{l}$ can be considered as excellent. In contrast, the Nova Biomedical biosensors showed a significant overestimation $(10 \%)$ that was seen in the same order of magnitude by Toffaletti [16]. The limitation of both biosensor methods is the high variability of the accuracy. A standard deviation of more than $13 \%$, as found in our investigation will present a true lactate value of $6 \mathrm{mmol} / \mathrm{l}$ between 4.4 and $7.6 \mathrm{mmol} / \mathrm{l}$ or even with higher difference. This result is in contrast to other investigations where the variability did not exceed $7 \%[2,10]$. The reason for the higher variability may be a result of our in vitro study design. Performing several series of measurements within a short period of time represents a much higher workload compared to other settings, although there are no restrictions from the biosensor manufacturers concerning the number of lactate measurements per unit time. A similar phenomenon with a glucose biosensor was shown by Cobbaert who found a variable decline in the recovery of the biosensors' enzymatic layers as the reason for this phenomenon [11].

Because most laboratory mistakes occur before testing [17], the major advantage of biosensor technology compared to photometric procedures results from the integration of the sensors into automatic analyzers and the complete lack of any sample preparation and dilution. The potential impact of the manual sample preparation was shown by the findings of the other two laboratory procedures initially included in this investigation. As the required sample dilution was insufficiently performed by the laboratory the inaccuracy in the Abbott and Analyticon, samples presented to be more than $30 \%$. Despite an internal laboratory quality control, these errors remained undetected until the results were unblinded at the end of the study. Although we could not determine the exact accuracy of both procedures, this shows the potential impact of preanalytical sources of errors that can only occur with a sophisticated manual sample preparation for standard laboratory procedures and not with the automatic biosensor devices.

When evaluating contemporary devices for lactate measurement, not only precision and accuracy are of importance but also the interchangeability and comparability of the different results obtained. One of the standard procedures of comparing different measurement methods is the model of logistic regression. When applying this to lactate analysis the results are often misleading because at low lactate concentrations the differences between methods are underestimated and at high lactate concentrations overestimated. Bland and Altman introduced a different method by calculating the mean (bias) and standard deviation (precision) of the differences between split sample determinations [6,7]. Especially when no reference method exists and the exact quantity of a value is not known as in lactate analysis, bias and precision provide adequate information on the interchangeability of two methods. Applying this to the results of the present investigation, even the comparability within the two biosensor methods of Chiron Diagnostics and Nova Biomedical appeared to be limited. Although the technical principle is identically in both devices, bias and precision indicated a very limited interchangeability that was in the same order of magnitude between the Chiron biosensor and Sigma pho- 
tometric procedures. As seen in the calculation of the accuracy above, the main reason for this reduced comparability is again the highly variable and therefore unpredictable accuracy of the biosensors investigated substantially limiting the value of a single determination.

\section{CONCLUSION}

In summary, the present data suggest that the accuracy of lactate determination with current biosensor methods (Chiron Diagnostics, Nova Biomedical) is acceptable for clinical purposes on average but at the same time highly variable limiting the value of a single determination and the comparability of results obtained with different procedures. The advantage of biosensor technology is the complete lack of any sample preparation and dilution minimizing the risk of preanalytical sources of errors what appeared to be the major potential drawback of photometric methods.

\section{APPENDIX}

\section{Principles of Lactate Measurement}

\section{Biosensor Technology}

\section{Principle}

A lactate sensitive enzymatic reaction combined with amperometric detection. The electron flow detected is linearly proportional to the lactate concentration.

Chiron Diagnostics (Medfield, MA, USA), NOVA Biomedical (Waltham, MA, USA)

Enzymatic reaction: Lactate $+\mathrm{O}_{2} \rightarrow$ (by Lactate Oxidase) $\rightarrow$ Pyruvate $+\mathrm{H}_{2} \mathrm{O}_{2}$

Amperometric detection: $\mathrm{H}_{2} \mathrm{O}_{2} \rightarrow$ (oxidation at constant current potential) $\rightarrow 2 \mathrm{H}^{+}+\mathrm{O}_{2}+2 \mathrm{e}^{-}$

\section{Photometric Laboratory Procedures}

Principle

A lactate sensitive enzymatic reaction combined with an indicator reaction. The spectro/photometrically detected indicator concentration is linearly proportional to the lactate concentration.

\section{Lactat PAP, Analyticon (Burbach, Germany)}

Enzymatic reaction: Lactate $+\mathrm{NAD} \rightarrow$ (by Lactate Oxidase) $\rightarrow$ Pyruvate $+\mathrm{H}_{2} \mathrm{O}_{2}$

Indicator reaction: $\mathrm{H}_{2} \mathrm{O}+\mathrm{p}$-Aminoantipyrin + Phenol $\rightarrow$ (by Peroxidase) $\rightarrow$ Chinonimin dye.

Procedure no. 735, Sigma Diagnostics (St. Louis, MO, USA)

Enzymatic reaction: Lactate $+\mathrm{NAD} \rightarrow$ (by Lactate oxidase) $\rightarrow$ Pyruvate $+\mathrm{H}_{2} \mathrm{O}_{2}$

Indicator reaction: chromogene precursors $+\mathrm{H}_{2} \mathrm{O}_{2} \rightarrow$ (by Peroxidase) $\rightarrow$ coloured dye.
TDxFLx, Abbott Laboratories (Irving, Tex, USA)

Enzymatic reaction: Lactate $+\mathrm{NAD} \rightarrow$ (by Lactate Oxidase) Pyruvate $+\mathrm{H}_{2} \mathrm{O}_{2}$

Indicator reaction: NAD + Monotetrazol dye $\rightarrow$ (by Diaphorase $) \rightarrow \mathrm{NAD}^{+}+$MTT-Formazan.

\section{ACKNOWLEDGEMENTS}

Supported by institutional funding except for the biosensor devices, that were supplied by Chiron Diagnostics, Fernwald, Germany, and Nova Biomedical, Rödermark, Germany.

\section{REFERENCES}

[1] Noordally O, Vincent JL. Evaluation of a new, rapid lactate analyzer in critical care. Intensive Care Med 1999; 25: 508-13.

[2] Slomovitz BM, Lavery RF, Tortella BJ, Siegel JH, Bachl BL, Ciccione A. Validation of a hand-held lactate device in determination of blood lactate in critically injured patients. Crit Care Med 1998; 26: $1523-28$.

[3] Boldt J, Kumle B, Suttner S, Haisch G. Point-of-care (POC) testing of lactate in the intensive care patient. Acta Anaesthesiol Scand 2001; 45: 194-9.

[4] Detry B, Nullens W, Cao ML, Frans A, Robert FA, Clerbaux T. Assessment of the lactate biosensor methodology. Eur Respir J 1998; 11: 183-7.

[5] Bénéteau-Burnat B, Bocque MC, Lorin A, Martin C, Vaubourdolle M. Evaluation of the blood gas analyzer Gem PREMIER 3000. Clin Chem Lab Med 2004; 42: 96-101.

[6] Bland JM Altman DG. Statistical methods for assessing agreement between two methods of clinical measurement. Lancet 1986; 81: 307-10.

[7] Bland JM, Altman DG. Comparing methods of measurements: why plotting difference against standard method is misleading. Lancet 1995; 346: 1085-7.

[8] Suistomaa M, Ruokonen E, Kari A, Takala J. Time-pattern of lactate and lactate to pyruvate ratio in the first 24 hours of intensive care emergency medicine. Shock 2000; 14: 8-12.

[9] Schmiechen NJ, Han C, Milzman DP. ED use of rapid lactate to evaluate patients with acute chest pain. Ann Emerg Med 1997; 30: 571-7.

[10] Brinkert W, Rommes JH, Bakker J. Lactate measurements in critically ill patients with a hand-held analyzer. Intensive Care Med 1999; 25: 966-9.

[11] Cobbaert C, Morales C, van Fessem M, Kemperman H. Precision, accuracy and linearity of radiometer EML 105 whole blood metabolite biosensors. Ann Clin Biochem 1999; 36: 730-8.

[12] Mantha S, Roizen MF, Fleisher LA, Thisted R, Foss J. Comparing methods of clinical measurement: reporting standards for Bland and Altman analysis. Anesth Analg 2000; 90: 593-602.

[13] Kruse JA, Carlson RW. Lactate measurement: plasma or blood? [Intensive Care Med 1990; 16: 1-2.

[14] De Keijzer MH, Brandts RW, Brans PG. Evaluation of a biosensor for the measurement of lactate in whole blood. Clin Biochem 1999; 32: $109-12$

[15] Smith EW, Skelton MS, Kremer DE, Pascoe DD, Gladden LB. Lactate distribution in the blood during progressive exercise. Med Sci Sports Exerc 1997; 29: 654-60.

[16] Toffaletti J, Hammes ME, Gray R, Lineberry B, Abrams B. Lactate measured in diluted and undiluted whole blood and plasma: comparison of methods and effect of hematocrit. Clin Chem 1992; 38: 2430-4.

[17] Lamb LS Jr, Parrish RS, Goran SF, Biel MH. Current nursing practice of point-of-care laboratory diagnostic testing in critical care units. Am J Crit Care 1995; 4: 429-34. 